\title{
Community Resilience in Face of Dystocia Deliveries: Case of Abbey Community in Ivory Coast
}

\author{
Mathieu Tchétché Obou ", Oscar Kouadio Koffi Bodoua \\ Department of Anthropology and Sociology, Alassane Ouattara University, Bouaké, Ivory Coast \\ Email address: \\ tchetcheom@gmail.com (M. T. Obou), bodoua.oscar@gmail.com (O. K. K. Bodoua) \\ ${ }^{*}$ Corresponding author
}

\section{To cite this article:}

Mathieu Tchétché Obou, Oscar Kouadio Koffi Bodoua. Community Resilience in Face of Dystocia Deliveries: Case of Abbey Community in Ivory Coast. World Journal of Public Health. Special Issue: Women, Health and Sustainable Development in Under Developed Country. Vol. 5, No. 1, 2020, pp. 17-23. doi: 10.11648/j.wjph.20200501.13

Received: January 8, 2020; Accepted: January 28, 2020; Published: February 14, 2020

\begin{abstract}
In Ivory Coast, the objectives of the sustainable development in health field seem to be upset by the frequency of dystocic deliveries. They contribute to the increase in the rate of maternity and mortality. In response to this concern, the community environment is developing resilience through various remedies. Like the different ethnic communities, each with its own options, the Abbey community is developing its own endogenous mechanisms. Also, how perceptions related to Caesarean section delivery determine the organization of resilience for women participants in this community. Through this questioning, the essential objective is to know the social perceptions related to the cesarean section, the limits of obstetric care offerings; and to present the attitudes of resilience with regard to the prognoses of dystocic births. The investigation has mobilized the qualitative approach in its case study tradition. It took place in the hospital and community settings of Oforidjé in Agboville and Azaguié Ahoua in Azaguié. On the basis of the network technique and the criterion of interruption of the collection at saturation of information, 67 people (qualitatively representative) were interviewed. The results reveal a social perception characterized by a view of the caesarean section as a factor of disillusionment for the participant or mother who has undergone it; a situation of offers of obstetrical care devoid of empathy for the pregnant and characterized by a change of scenery of the latter in the delivery room. Resilience in this community is characterized by perinatal care in the form of ritual and treatment with plants, bark and roots.
\end{abstract}

Keywords: Ivory Coast, Abbey Community, Dystocic Deliveries, Resilience, Caesarean Section

\section{Introduction}

In the field of health, faced with the problem of sustainable development dystocic deliveries remain an obstetrical concern. This type of delivery is characterized by complications such as hemorrhage, anemia, eclampsia, overweight fetus, narrowed mother's pelvis, and hypertension for which the recommended route of delivery is caesarean section. In fact, caesarean section is a surgical act that consists of extracting the child from his mother's womb by incision from the uterus.

According to the WHO, every day approximately 810 women died from preventable causes related to pregnancy and childbirth. Between 2000 and 2017, the maternal mortality ratio (MMR, number of maternal deaths per 100,000 live births) dropped by about $38 \%$ worldwide. $94 \%$ of all maternal deaths occur in low and lower middle-income countries [1]. This justifies Objective 3 of the Sustainable development goals (SDG) stipulating that By 2030, ensure universal access to sexual and reproductive health-care services, including for family planning, information and education, and the integration of reproductive health into national strategies and programs [2].

In Ivory Coast, the desire to reduce maternal mortality was demonstrated through the recommendation of the caesarean section and the assumption of the cost of the kit of the operation. Despite this provision, the maternal mortality rate of 597 deaths per 100,000 live births in 2011 [3] increased to 645 deaths per 100,000 live births in 2015 from an average of 546 for sub-Saharan Africa [4]. Among the death factors, caesarean section remains the main cause of the increase in the rate mentioned in public opinion. 
Rightly or wrongly, the populations incriminate the financial and material conditions of his practice despite the assurance by the authorities of the existence of cheaper conditions of practice of this surgery.

Through this study, it is a question of understanding how perceptions related to caesarean section delivery determine the community's organization of resilience in favor of female participants. Indeed, the concept of resilience is of mechanical origin and borrowed by the social sciences from the physics of materials. In mechanics, it is defined as the ability to absorb cynetic energy without breaking [5]. It brings back the social dimension to the ability, on the one hand, of individuals placed in unfavorable circumstances to "get by" [6] and, on the other hand, of actors to lead "a good life" through the ways in which they manage to cope with change [7]. On the other hand, it is the ability to succeed, to live and to develop positively, in a socially acceptable way, despite destabilizing events [8]. It is also the universal human capacity to face, overcome and even be transformed from the adversities of life [9]. This includes the ability to succeed despite stress or adversity [10]. It is also used to describe the resistance of the child or adult in case of precariousness [11].

In the field of childbirth and gynecological care, resilience refers to a mother's ability to overcome the problems associated with the intervention and their impact on the quality of her social life. To preserve this quality of life, some United Nations organizations committed in 2002 to promote non-clinical actions to reduce the number of caesarean sections in a special research program, development and training in human reproduction research [12]. In spite of these efforts, the culture of multipartite in African community circles makes people fear the consequences of caesarean sections and hemorrhagic complications. Many concepts are therefore developed around modern management approaches and endogenous solutions for monitoring pregnancies and births.

In the case of the Abbeys of Ivory Coast, the resilience initiative is based on ancestral knowledge and the critical view of medical care. As well as, the present study questions the perceptions of dystocic birth sufferings in the Abbeys; presents the characteristics of the demand for eutocic birth within this people in the face of the limits of obstetric care offer; and elucidates the attitudes of resilience in the light of the prognostic of dystocic births.

\section{Method}

\subsection{Investigation Sites and Population}

The study has been carried out sequentially since January 2012 in Azaguié Ahoua in the town of Azaguié and Oforidjé in the town of Agboville with mothers who have undergone caesarean or not, pregnant women and matrons in the Abbey community. It has also expanded to the Agboville Regional Hospital and the Azaguié Urban Health Centre to gather the views of healthcare providers. It should be noted that the
Abbeys are a people of the South of Ivory Coast. They are located in the Agnéby Tiassa region. The main towns are Agboville, Azaguié and Grand-Morié.

\subsection{Data Collection Technique and Theory of Analysis}

The survey is qualitative in nature and is part of the case study tradition. In this context, the study reports on the experiences of the respondents in relation to the social demand for normal (eutocic) childbirth. On the basis of the decision to discontinue investigations on the basis of information saturation, the investigations involved 20 mothers who had caesarean sections on indication during pregnancy and others at childbirth, including those who had suffered minor complications; 06 pregnant women; 04 matrons; 07 spouses of caesarean section mothers, 15 companions of mothers who gave birth by caesarean or not, 03 community leaders, 12 officers and practitioners of caesarean sections at the Azaguié health training and at the Agboville regional hospital. In total, investigations were stopped after semidirect interviews with 67 people.

The technique of collecting data from identified persons is the network or snowball technique. It lends itself better to the context of this study. It consisted for the members of the community, to direct the investigators to the persons carrying the information sought. These are the patients surveyed and others who were aware of the problem delivery event, including the patient's family members, caregivers in the community care of the mother and child.

The units of observation are as follows: the age, the family situation, the uterus situation, the event of the first caesarean section or complication and the option faced with a next risk related to childbirth.

Among the women surveyed, the minimum age is 16 and goes beyond 50. It is divided between adolescent and young mothers (under 21), active mothers (between 21 and 44) and menopausal mothers (at least 45 years according to medical logic). These women are recruited from among pregnant women, C-sections or not, attendants and matrons. Thus, active mothers account for $80 \%$; adolescent girls and young people for $15 \%$ and menopause for $5 \%$. Of the women interviewed, $60 \%$ live maritally and $20 \%$ are divorced or experiencing marital difficulties. In addition, $70 \%$ of caesarean sections are single-scarred and 30\% doubly healed.

The deepening of the results necessitated the mobilization of the theory of resilience allowed women to avoid Caesarean section [5-11].

\section{Result}

The results of the study are structured around three main points. The first relates to perceptions of dystocic birth pain The second concerns the limits of obstetrical care offered in response to the demand for eutocic birth. And the third point deals with Attitudes of resilience to dystocic Birth Prognosis. 


\subsection{Perceptions of Dystocic Birth Pain Among Abbey Participants}

The conception of normal childbirth in the Abbeys is that which is carried out by the low way without causing hemorrhage and where the mother and the child leave unharmed. On this basis, community perception of dystocic birth sufferings among Abbey participants suggests the bitterness caused by physical and moral injuries.

\subsubsection{Bitterness of Physical Injury}

The idea of having a scar as a result of a C-section is perceived by the populations interviewed as a traumatic situation. For women, she makes disappear the pleasure of making a child. They think that the next $\mathrm{C}$-section at the party can be fatal. It is a warning to them that we must take seriously. From then on, they are perplexed about a full experience of sexuality after a cesarean section. This perplexity is explained by recurring questions in the opinion of the women interviewed. One says, "After how long can a caesarean section woman go with her husband?". This question raises the problem of the sexual future of women. To justify it, the women evoke the stress caused by the spouse's willingness to realize the resumption of sexual activity after the caesarean section by a new pregnancy. This is reflected in the second question that emerges among the women interviewed. It says, "Why can some have a pregnancy two years after surgery and others not?".

The men interviewed also direct their perception towards the discouragement that can be caused by the sight of a caesarean section scar in a new female conquest. A young man who experienced Caesarean section during the delivery of his girlfriend, claims to be charged with the responsibility for the scar of the girl. He explains that this is manifested by the accusing remarks of the daughter and her parents and especially by recurring acts of jealousy. As with women, almost all of the men interviewed believe that caesarean section reduces the prognosis of having children with the woman. It is now considered to be a person with a disability. Thus, when it evokes any pain during or after sexual intercourse, they incriminate the practice of caesarean section and the solution they propose is that of taking a second wife. A 52-year-old spouse in Oforije, with only one daughter with his wife following a C-section, says: "I cannot have one child; the daughter cannot manage the family according to custom; boys are needed; except my wife is 46 years old; with the Csection, she can no longer have children; I have to take a second wife...".

This situation is a source of permanent conflict in couples because if custom allows it, the woman attached to her fulfilment and her rights conferred by the state, is opposed to this kind of decision. This often results in separation when polygamy is refused by the woman. Almost all of the mothers interviewed in a divorce situation or experiencing marital difficulties as a result of the practice of caesarean section during their childbirth, highlight the link between their caesarean section and the palaver that led to their divorce or marital difficulties.

\subsubsection{Bitterness of Moral Wounds}

Moral injuries are characterized by community accusations that are believed to be the basis for caesarean section and other complications. They are socio-cultural in nature. For the women interviewed in the Abbey community, they appear important and are characterized by several packages. This is the violation of the customary rules of marriage. It involves a lack of respect for the in-laws or spouses, marital infidelity, failure to observe the rites of atonement in the event of transgression, and the pregnant woman "washing yourself out late at night", poor eating habits, poor use of language against the elderly (curses, disputes), and noncompliance with community practices in the event of pregnancy.

Perceptions of the circumstances of dystocia during childbirth are linked to these packages. In fact, it is first and foremost the perception of childbirth difficulties by women workers as being related to probable marital infidelity. Investigations show that in the Abbeys, when the pregnancy was contracted as a result of the mother's marital infidelity, it can lead to death by caesarean section or hemorrhage. To avoid the loss of the child and/or mother, the mother is subjected to a confession ritual during childbirth. The medical actors interviewed perceive the submission to this ritual by the attendants or the matron in a state of suffering, as additional humiliation and violence. It has been said repeatedly in their opinion that they "hinder our work", "they want us to work". These statements show the reluctance of medical practitioners to solicit the contribution of accompanying community nurses.

Second, women perceive Caesarean section delivery as a personal frustration in their willingness to give life because of the rumors of infidelity when the situation arises. They also see it as "a failure", as the expression of "inability to deliver like others". It is a state of childbirth which is still perceived as the result of a mystical attack that did not succeed. The women interviewed refer to alleged perpetrators as a parent, step-in-law or third party.

In addition, caesarean section is perceived as a source of expense incurred by medical personnel. The statements collected from the medical actors indicate recurrent refusals of the participants in case of reference for cesarean section. This refusal is related to the lack of financial means and the belief in traditional delivery solutions. Regarding the cost, the populations of women questioned deny the effective practice of the gratuitousness of the caesarean section proclaimed by the government. A mother who has had a C-section twice reports, "If you don't pay what they ask you, they will leave you there; for me it's the family who paid the first time; the second time my husband took a credit...".

Finally, the death of the fetus despite the caesarean section is perceived by the Abbey populations as the major psychological shock of the parturient. This comment about a woman having a caesarean section seems to express this pain 
by saying, "Not only have you been torn apart, but your child did not stay". This corroborates a specialist's explanation: "What creates shock is when the child is stillborn while she sees herself healed for him; in other circumstances, the parturient hears the cry of her child; when she sees it, she then feels comforted by the caesarean section; postoperative pain: this is no longer her problem; but when there is a case of stillbirth, the shock is very difficult for her; this can even lead to insanity...".

In total, the set of perceptions evoked is fed by the popular imagination of Abbey. Nevertheless, some limitations in the scope of obstetrical care services contribute to the promotion of caesarean section births.

\subsection{Limits on Offers of Obstetrical care in Response to the Request for Eutocic Birth}

This point highlights the inadequacies of the medical supervision and follow-up and the medical factors for the indication of the caesarean section.

\subsubsection{Inadequate Supervision and Medical Follow-up During Pregnancy}

Most mothers who have undergone caesarean section against their will and the matrons denounce a cultural difference between them and the players in the medical system. Mothers deplore the fact that all the advice given to them during prenatal consultations systematically refutes ancestral practices. The words of a multipart mother are evocative. She says, "Everything is imposed on us; even the midwives of Abbey origin do not want us to do the traditional treatments..." Among the matrons, those who are recognized as community health workers claim to introduce in the protocol of management of childbirth, traditional solutions that avoid caesarean section to women. However, the matrons who come as attendants on the day of delivery claim to be rejected by the midwives. One says, "we have drugs so that we don't rip up the woman, but people don't want to listen to us".

Other factors are related to the care behaviors of health workers. To be clear, almost all community informants have revealed that women are not really in the long term and are given birth. They deplore injections to say "speed up work". They question midwives' work experience by saying that they "do not have good knowledge of delivery methods". The populations are surprised by the systematic indication of caesarean section in case of a small suspicion of anomaly. They conclude with the question of "whether there is profit for the staff who refer the patient."

Women who have experienced childbirth also evoke the dislocation caused by the delivery rooms. All claim that the fear of the delivery room device frightens any woman especially the primiparous. They cite as their main consequence the blockage of contraction when there is no one in the room they can trust; A 26-year-old mother with a caesarean section reports: "When I was made to sleep on the hospital bed, it cuts off my breathing... I couldn't push anymore; the midwife was yelling at me, then I didn't see the rest... when I woke up, I saw that I had an operation".

In total, the lack of communication about the stages of care during childbirth and the refusal to accompany the matron or to use its medications to avoid complications makes the caesarean section inevitable.

\subsubsection{The Questioning of Medical Factors for Caesarean Section Indication}

Among the C-section mothers in the population of surveyed mothers, $80 \%$ were reported on delivery for the first intervention and $20 \%$ on pregnancy after having undergone a first operation.

The medical factors for the indication of the caesarean section vary from woman to woman. These are: age, pregnancy rank, pelvic morphology and fetal weight. All these factors are often punctuated by a fragile state of morbidity in the mother. These include malaria in pregnancy, malnutrition, anemia and hemorrhages during pregnancy or childbirth.

Clearly for medical actors, the prescription of caesarean section is the most obvious, especially for primary adolescent girls and multiparas over the age of 44. Among teenage girls with a first pregnancy, children are overweight due to a lack of adherence to medical instructions for some and for smaller pelvis reasons for others. In multiparas over 44 years of age, the ranking of pregnancy is the main cause. Practitioners reveal that from the fourth pregnancy, the chances of normal childbirth decrease and the risks of caesarean section increase. They say that the trend in rural areas is to go beyond three children per woman. For all the participants, the caesarean section becomes inevitable in the face of a poly-myomateous uterus (cysts, myomas, fibroids); a scar uterus (history of caesarean sections); a presentation of the fetal seat or transverse; Fetus overweight (more than $3.5 \mathrm{Kg}$ ); high blood pressure, and so on.

The women interviewed agreed that these objective factors are known in the community. However, they reveal that the main factor is rather the rejection of community collaboration in providing solutions.

Thus, Faced with the recurrence of caesarean section despite submission to medical solutions, a massive use is made of community care as an attitude of resilience.

\subsection{Attitudes of Resilience to the Need for Eutocic Birth}

Resilience in the Abbey community is characterized by actions of marginal protection of women during pregnancy or during childbirth. It's behavioral resilience and therapeutic resilience. This is done with the accompaniment of the whole family is matrons.

\subsubsection{Behavioral Resilience}

Behavioral resilience is preventive in nature. In the popular imagination of Abbey, respect for uses and customs remains a factor favoring fertility and good childbirth. This requires adherence to recommended community habits and practices for women and girls facing pregnancy. Thus different treatments are administered to the parturient to 
avoid any unfortunate consequences.

The first disposition is not to declare the pregnancy to his circumposing before his appearance except to the very close people unsuspected of mystical attack. The matrons interviewed indicate that it is possible to talk about pregnancy from the 4 th and 5 th months when we see the belly increasing in volume.

The second provision is that as soon as the delay is noted, the woman must make peace around her. If there were conflicts between her and her spouse or step-parents, she must work to resolve them. The matrons say that such a disposition reassures the spirit of the fetus who wants to be convinced to be born in a good family environment. A matron in Oforidjé reports, "If there is too much talk, the child returns...".

The third provision relates to the organization of the parturient bath throughout the pregnancy. According to the matrons, the parturient must not wash herself in the open air beyond 18 hours. They report that if this is the habit of the pregnant woman, evil spirits can disrupt the good evolution of pregnancy and childbirth.

The fourth provision consists in consolidating the behaviors listed by treatments having the same function. These are medicines for ingestion, fumigation (steam bath) and drinking solutions based on plants, bark and roots. The medicine is said in Abbey «Kpéh». The main drug is made against general fatigue, the complicated form of which is literally called "stomach wound" and against nerve problems according to them. The treatment is called in Abbey "Opubè Kpéh" or "Kalè Kpéh". It is a care product made from dried and ground bark. It is used by preparing a broth with the powder to which at least five (05) African peppers are added, either consumed in drinkable solution or ingested. The other drug is the "Chipèh Kpé" against the effects of anger on childbirth. It manifests itself in the manifestation of high blood pressure in the mother and prevents proper work. In the same register, the drug "Ofuagnon Kpéh» is used against the wrong positioning of the child which the community links with its refusal to be born in a poorly breast environment. Both drugs are within reach during childbirth and are administered when the above-mentioned difficulties arise at work.

\subsubsection{Therapeutic Resilience}

Therapeutic resilience is characterized by traditional treatments during pregnancy and those occurring during childbirth in the event of signs of complications. About treatments during pregnancy, several therapies are offered. They start from the first trimester of pregnancy to delivery in the ninth month.

"Adonèh pkéh" powder. "Adonèh" refers to the malformation of the head or/or limbs. The pregnant woman uses it from the 3rd month until the time of delivery. It is a powder made from ground bark, mixed with African pepper, warmed and consumed as an ingested solution or ${ }^{2}$ and then crushed to apply it on the stomach. According to the participants and matrons, it secures the development of the fetus by making it possible to avoid birth defects.

Kaolin "Efiéh pkéh". "Efieh" is the meaning of pelvic bone. "Efieh pkéh" means "bone medicine". According to the Abbey Community Nosography, this drug, if applied during the period of pregnancy, helps to adjust the uterine bones of the pregnant woman's pelvis. According to the testimony of interviewees, it is effective against the narrowed basins. The comments that come out of the surveys are "it opens the pelvis to get the baby out" "it also treats the large fetuses". It is recommended to drink three (3) times a day, to massage your waist and lower stomach regularly. If the case of narrowed pelvis is not obvious, this medicine is used from the 8th month onwards to prevent cases of large fetal evacuation (Above $3.5 \mathrm{Kg}$ ).

The "Adoh pkéh" ball. "Adoh" is the umbilical cord translation. This medicine is used to prevent the umbilical cord from choking the child during childbirth. The pregnant woman uses it before the end of the pregnancy. After eight (08) months, when the ultrasound reveals that the child has taken the cord, the caregiver begins to drink it and apply it to the body.

The «mijih pkéh» pellet. «mijih» means water. It is done by urinary discharge. The pregnant woman uses it to reassure herself of the quality of the placental fluid. Regular treatment with this medicine avoid stillbirths, premature births and low birth weight (less than $2.5 \mathrm{Kg}$ ).

The «Opulah pkéh» pellet. «Opulah» means hot belly. This product is generally used against stomach heat which is not conducive to the proper development of the fetus. This is literally called Hot Stomach Disease. Four (04) or five (05) months later, pregnancy may fall due to lack of treatment. The woman at the beginning of the pregnancy must drink it and apply it on her stomach when she notices the beginning of the pregnancy and feels this heat. The serious (chronic) form of the "Opulah" is called " $n$ 'gborugboh". The kaolin «Pioh Kpéh». «Pioh» is the rectum. «Pioh Kpéh» is therefore the disease of the rectum. In other words, it's the treatment of hemorrhoid. This treatment is done to prevent the manifestation of this disease from obstructing the woman's deliverance on the day of delivery. He leaves from the third month until the end of the pregnancy.

The sheet of "Mpiahu Kpéh". "Mpiahu" means bleeding. It's a leaf that macerates in the mouth when the bleeding starts during childbirth. During pregnancy, the broth of the fresh leaves of this plant is used to treat cases of anemia of the pregnant woman in the community.

The following figures show some aspects of the drugs used.
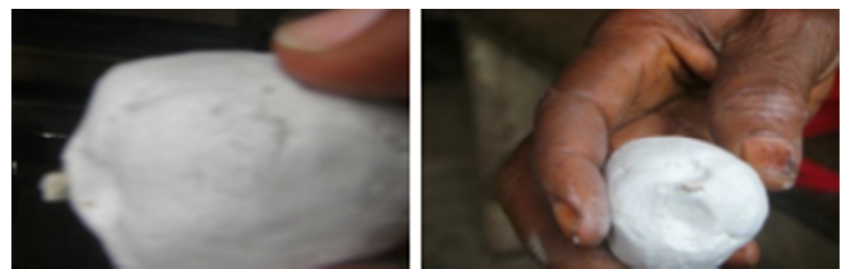

Figure 1. A view of the "Efiéh pkéh" kaolin By Oscar Bodoua, AzaguiéAhoua, investigation of January 2012. 

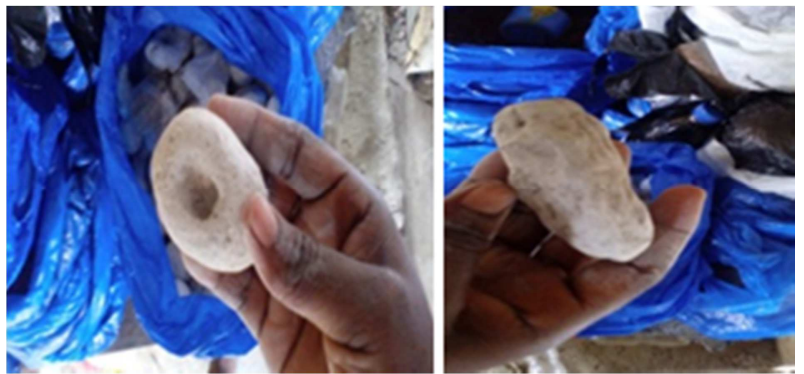

Figure 2. A view of the "Pioh Kpéh" kaolin and it's conservation mode By Mathieu Tchétché, Oforidjé, investigation of August 2017.

\section{Discussion}

Three strong ideas are the subject of discussion. The first idea relates to perceptions, the second concerns the social valorization of the problems of poverty following caesarean sections.

\subsection{Caesarean Section as the Manifestation of Conflicts with Invisible Forces}

The results of the study show that the perceptions of Caesarean sections during childbirth are largely related to non-medical circumstances. These are characterized by customary rules of respect for the in-laws or spouses, and faithfulness to the latter. It reveals a link to the elders and their blessings. They are therefore the guarantors of compliance with the various provisions and the main players in the compensation of lump sums. When the repair is not done, the health of the parturient may suffer during pregnancy. The caesarean section in is the visible consequence and death, the supreme sanction. For these situations to happen unfortunately, we need an extension of the resentments of the elders to the spirits of the ancestors face to the violation of the rules for mobilizing the chances of childbirth. Thus, the safeguard of the well-being of the woman, dependent on the ancestors, the blessing of the elders of the clan and the scrupulous observance of prohibitions by both spouses [13].

As an example, among the Mossi of Burkina Faso, procreation is linked to penetration a spirit called "kinkirga" in the woman's womb at the time she has sex with her husband [14]. The spirit is therefore an omnipresent force at the moment of conception, of pregnancy and childbirth that should be avoided by upsetting by behaviors deemed unworthy in the community.

\subsection{Caesarean Section as an Attack on the Hope of the Social Valorization of Women}

It is right that women who have undergone Caesarean section and who have to limit the number of births

are bruised. Indeed, in Abbey society, it is a privilege for the woman to be nurtured by the entourage and book him an official release party with his newborn after 03 months. This disposition is made as many times as the woman childbearing.

This is all the more important since pregnancy is a crucial event in a woman's life [15]. In Madagascar, it is called a "moment of hope" because of its value. But, it is also, a "moment of peril" with regard to the risk of death in the mother and in the child, so well that during pregnancy, the women who are at the party show no joy in their actions, nor in their words, nor on the features of their faces.

This is why diseases attributed to supernatural factors (convulsions, delusions, follies), to transgression of a taboo or to the wrong fate (infertility, abortion), can be treated only by the traditional medicine and the use of healers or soothsayers and in no case by medicine [16]. Also, the intervention of traditional healers, matrons or religious leaders, in case of obstetrical complication, is a common alternative when using care, including in urban environment [17].

\subsection{Caesarean Section as a Brake on Family Prosperity}

The woman in the country Abbey has the role of perpetuating the lineage. By making several children she contributes to the prosperity of the family on several levels. At the level of boys, it is the latter who ensure inheritance. When they have a social success, their mother is automatically haloed. At the level of girls, their childbirth is also guarantee of material and financial ease both through the benefits of her future marriage but also of her paid work when has academic success and university.

On the contrary, the woman who cannot offer as many children as she wishes for her family for her prosperity is likely to end his days in misery. It is true that family planning solutions are offered to Abbeys women and beyond, to all rural women in Côte d'Ivoire and Africa. But when the children have not yet reached their desired quota of children or have not undergone of complicated childbirth, or do not risk it, they are not interested in it. This fact is corroborated by the experience of the Mossi families of Burkina Faso where reproduction is «the assurance of the prosperity of the family" [14].

Thus, the number of children and gender variation among children is a major asset for the enrichment of the family.

\section{Conclusion}

At the end of this work, it is clear that the perceptions of caesarean section within the Abbey community of Côte d'Ivoire reveal women who are physically and morally diminished. They deplore the loss of the pleasure of giving birth normally and of a couple's life without resentment of the spouse. The prevalence of caesarean section is also seen as a symbol of rejection of traditional fertility values by community members. Similarly, it is seen as an expression of contempt for modern medicine in relation to traditional medicines used by women during pregnancy and childbirth.

This set of perceptions commands Abbeys matrons and healers to promote resilient social behaviors and therapeutic attitudes. They do it with rural women in this community and other urban Abbeys women or not, in search of children in normal childbirth conditions. Aside from the demands of 
community values that mobilize the blessings of parents and social elders, Abbey community resilience involves the use of kaolin, decoction, fumigation and ingestion of medicinal plants whose therapeutic virtues are recognized in the community.

Given the persistence of resilience solutions due to the community rejection of the caesarean section, it is important to take a number of actions in the health districts. The main act would be to establish Community monitoring as a model ideal. This implies the effective clarification and practice of the directives on community involvement in basic health care To do this, it is necessary to maintain the dialogue between the actors of modern therapeutic approaches and those of traditional therapeutic approaches to include in the medical system the local approaches with solutions for health problems deemed to devalue within communities.

\section{Acknowledgements}

Thanks to the managers of the Word Journal of Public Health for the call for papers. Our infinite gratitude goes to the lead guest editor Meless Simeon Akmel for the solicitation, to the members of Abbey community and medical professionals in Agboville and Azaguié who made possible the realization of this research.

Merci aux responsables de Word Journal of Public Health pour l'appel à contribution. Notre infinie gratitude va à l'éditeur invité principal Meless Simeon Akmel pour la sollicitation, aux membres de la communauté Abbey et aux professionnels médicaux d'Agboville et d'Azaguié qui ont rendu possible la réalisation de cette recherche.

\section{References}

[1] WHO (2019). Maternal mortality, online, https://www.who.int/news-room/fact-sheets/detail/maternalmortality, available the $26^{\text {th }} 2019$.

[2] UN Women (2015). SDG 3: Ensure healthy lives and promote well-being for all at all ages, Online, https://www.unwomen.org/en/news/in-focus/women-and-thesdgs/sdg-3-good-health-well-being, available the $26^{\text {th }}$ December December2019.

[3] RCI (2013). Enquête démographique et de santé et à indicateur multiples (EDS-MICS) 2011-2012, Abidjan, pp324-325.

[4] RCI (2016). Plan National De Développement Sanitaire (PNDS) 2016-2020, 88p.
[5] M. Anaut (2005). Le concept de résilience et ses implications cliniques, Revue recherche en soins infirmiers, no 82, pp 4-11.

[6] M. Rutter (1985). Resilience in the face of adversity: protective factors and resilience to psychiatric disorder. Britanique Journal Psychiatry, 147, 598-611.

[7] A. P. Hall and M. Lamont (2013). Social resilience in the Neoliberal Era, Cambridge, CambridgeUniversity Press, 396p.

[8] M. Manciaux (2001). La résilience: un regard qui fait vivre, 10 TOM 395, pp 321-330, Online https://www.cairn.info/revue-etudes-2001-10-page-321.htm, available the $29^{\mathrm{e}}$ december 2019 .

[9] E. H. Grotberg (1996). The International Resilience Project Findings from the Research and the Effectiveness of Interventions, online, https://files.eric.ed.gov/fulltext/ED419584.pdf, available the $13^{\text {rd }}$ decembre 2019.

[10] S. Vanistendael (2004). La résilience ou le réalisme de l'espérance. Blessé mais pas vaincu, les cahiers du Bureau international Catholique de L'Enfance, Genève, online, www.education-sante-ra.org/publications/2004/resilience.pdf, available the $11^{\text {th }}$ september 2015 .

[11] M. Phaneuf (2013). La résilience: concept abstrait ou pratique de vie, online, http//:www.prendresoin.org/wpcontent/uploads/2013/01/la-resilience-1-pdf, available the $12^{\text {th }}$ september 2015.

[12] OMS (2012). Programme spécial PNUD/UNFPA/OMS/Banque mondiale de Recherche, de Développement et de Formation à la Recherche en Reproduction humaine. Événements marquants de l'année 2011. Online, https://apps.who.int/iris/handle/10665/70937. $29 \mathrm{p}$, available the $30^{\text {th }}$ decembre 2019.

[13] M. L-Guimera (1977). La classification Evuzok des maladies, première partie, journal des africanistes, 47, 1 (1977), pp 9-51.

[14] K. R. Ouédraogo (1985). Femme et procréation: Etude monographique de la société traditionnelle mossie au Burkina Faso, école ouvrière supérieure, Institut Supérieur des Sciences Humaines Appliquées, 1080 Bruxelles.

[15] B. Rovolodomanga (1991). Pour la santé et la beauté de l'enfant à naître, in Grossesse et petite enfance en Afrique Noire et à Madagascar, édition Harmattan.

[16] R. O. Tchicaya, A. Kouvouama and J.-P. Missié (2014). Sociétés en mutation dans l'Afrique contemporaine: dynamiques locales, dynamiques globales, Karthala, p206.

[17] A. Dumont (2014). Comment réduire la mortalité maternelle dans les pays du Sud ? in Le Monde de Gynécologie sans frontière, Journal semestriel GSF, No 1 Octobre 2014. 